\title{
RECENSIÓN
}

\section{ATLAS OF MORTALITY IN EUROPE SUBNATIONAL PATTERNS 1980/1981 AND 1990/1991}

WHO Regional Publications

European Series N1 75

1997, ISBN 9289013397

245 páginas

Recensión: Fernando Villar Álvarez. Subdirección General de Epidemiología, Promoción y Educación para la Salud. Dirección General de Salud Pública. Ministerio de Sanidad y Consumo.

Esta publicación de la Oficina Regional para Europa de la Organización Mundial de la Salud recoge el resultado de un importante esfuerzo coordinado llevado a cabo por numerosas organizaciones nacionales e internacionales. Nace fundamentalmente fruto de la colaboración de cuatro instituciones: el World Health Organization European Centre for Environment and Health, el United Nations Economic Commission for Europe y el Central Bureau of Statistics y el National Institute of Public Health and the Environment de Holanda, que contaron con la participación activa de los institutos de estadística de los países europeos y de la Oficina Estadística de las Comunidades Europeas (EUROSTAT).

El atlas, junto con una serie de datos demográficos básicos, presenta las tasas de mortalidad nacionales de los países de la región europea de la Organización Mundial de la Salud, según las principales causas de muerte. Además proporciona los datos correspondientes a las regiones de cada país y muestra los cambios en la mortalidad regional entre los años 1980/1981 y 1990/1991. Todos los datos se presentan desagregados según el género. La mortalidad se expresa en forma de tasas ajustadas por edad empleando el método directo y utilizando como estándar la población europea. Esto nos permite apreciar las diferencias de mortalidad debidas a distintas causas en la región europea, indicando las áreas geográficas donde es necesario profundizar en el estudio para determinar los motivos que pueden estar justificando estas diferencias y, por tanto, mostrándonos la forma más apropiada de actuación para reducir estas diferencias. Todo ello permite una rápida y fácil comparación de los problemas de salud en Europa.

Este libro consta de cuatro capítulos y seis apéndices que se distribuyeron a lo largo de 245 páginas. Fl primer capítulo explica el origen y los objetivos del Atlas. En los capítulos segundo y tercero se exponen las cuestiones metodológicas sobre la recogida de los datos, su calidad, análisis e interpretación. El capítulo cuarto se dedica en exclusiva a los mapas y gráficos de barras. Se presentan mapas y gráficos, entre otros, para el conjunto de todas las causas de muerte, para las enfermedades infecciosas, tumores malignos, tumor maligno de colón y recto, tumor maligno de traquea, bronquios y pulmón, cáncer de mama, leucemia, enfermedades del aparato circulatorio, enfermedad isquémica del corazón, enfermedad cerebrovascular, enfermedades del aparato res- 
piratorio, enfermedades del aparato digestivo, enfermedades del aparato genitourinario y causas externas de traumatismos y envenenamientos.

Uno de los atractivos de este libro es que todos los datos se muestran a través de mapas y gráficos de barras. Hay que resaltar la magnífica presentación de los mapas, con una excelente calidad gráfica y cromática, y que constituyen la parte principal del libro, haciendo honor a su título. Los mapas nos permiten, con una ojeada, apreciar de forma simple, clara, agradable, completa e inteligible como se distribuyen geográficamente los principales problemas de salud en Europa.

No espere el lector encontrar en este libro interpretaciones, comentarios, valoraciones o conclusiones de los datos que se presentan. Se trata de un libro estrictamente descriptivo, lo que no es poco, que muestra de forma gráfica y clara la situación de mortalidad por distintas causas de muerte en Eu- ropa. La interpretación y valoración de los datos queda, por tanto, para el lector.

Hubiera sido muy interesante, como ocurre en otras publicaciones de la OMS, que el Atlas se acompañará de su correspondiente versión electrónica, mediante la inclusión de un disquete o CD-ROM, para permitir un más fácil acceso y uso de la copiosa información que contiene.

Por todo lo que se acaba de comentar, este Atlas puede resultar de gran interés como libro de consulta y estudio para los planificadores y gestores, para los investigadores y los docentes de ciencias de la salud. Permite disponer de una potente herramienta para el estudio de la epidemiología descriptiva de la mortalidad en Europa en un momento dado y de su evolución durante un decenio, para detectar zonas geográficas con un exceso o defecto de mortalidad y para generar hipótesis etiológicas que orienten investigaciones futuras. 